\title{
Soup burns and the roles played by viscosity, solid constituents, epidermal thickness and clothing
}

\section{Hunter N. Hickman, Jared T. Powell and Tony K. Kerzmann}

\author{
Engineering Department, \\ Robert Morris University, \\ Moon Township, PA 15108, USA \\ Email: hnhst311@mail.rmu.edu \\ Email: jtpst262@mail.rmu.edu \\ Email: tkerzmann@pitt.edu
}

\section{Alexandra C. Cox and Gavin A. Buxton*}

Science Department,

Robert Morris University,

Moon Township, PA 15108, USA

Email: accst7@mail.rmu.edu

Email: buxton@rmu.edu

*Corresponding author

\section{Carl Ross}

\author{
Nursing Department, \\ Robert Morris University, \\ Moon Township, PA 15108, USA \\ Email: ross@rmu.edu
}

\begin{abstract}
One of the many concerns for parents of young children and caregivers of geriatric patients is the potential for scalds. Skin tissue can be thinner and more susceptible to scalds, and the accidental spilling of hot soups can be a common source of burns. The type of soup, however, may not elicit any such misgivings. Here, we elucidate the effects of reduced skin thickness on the severity of soup burns. In particular, we compare the two most popular types of soups (tomato and chicken noodle) along with hot water, to identify the roles that the viscosity and solid constituents in these different soups may play in the severity of the burn. We find that the more solid constituents in the chicken noodle soup, in particular, prevents the soup from flowing from the skin, which increases the time of exposure to elevated temperatures, and therefore increases the severity of the burn.
\end{abstract}

Keywords: soup burns; scalds; heat transfer; viscosity; Pennes bioheat equation.

Reference to this paper should be made as follows: Hickman, H.N., Powell, J.T., Kerzmann, T.K.., Cox, A.C., Buxton, G.A. and Ross, C. (2019) 'Soup burns and the roles played by viscosity, solid constituents, epidermal thickness and clothing', Int. J. Computational Medicine and Healthcare, Vol. 1, No. 1, pp.73-87. 
Biographical notes: Hunter N. Hickman is a recent graduate of the BSc Engineering Program at the Robert Morris University.

Jared T. Powell is a recent graduate of the BSc Engineering Program at the Robert Morris University.

Tony K. Kerzmann has recently moved to the University of Pittsburgh where he is an Associate Professor at the Swanson School of Engineering. He received his MSc in Mechanical Engineering in 2007 and his $\mathrm{PhD}$ in Mechanical Engineering in 2010, both at the University of Pittsburgh.

Alexandra C. Cox is a recent graduate from the BSc Biology Program at the Robert Morris University.

Gavin A. Buxton is a Professor of Physics at the Robert Morris University. He received an MSCi in Engineering Physics in 1998 and a $\mathrm{PhD}$ in Computational Physics in 2001 from the Sheffield Hallam University, UK.

Carl Ross is a University Professor of Nursing at the Robert Morris University. He received his BSc in Animal Science in 1979 at the Pennsylvania State University, BSc in Nursing at the University of Pittsburgh in 1985, MSc in Nursing in 1994 and PhD in Transcultural Nursing in 2000, both at Duquesne University.

\section{Introduction}

Not only are burns potentially one of the most painful injuries, worsening with time as the skin contracts and the nerves regenerate, but they can also be one of the most preventable. In particular, we look at scalds from hot liquids and soups, in the hope of highlighting the physical parameters that influence scalds the most. It is our hope that this might help to establish guidelines for care givers of young and elderly people, who are the most susceptible to this kind of burn. Factors which affect the severity of scalding are the temperature, the viscosity, and the heterogeneity, or the 'chunkiness', of the fluid. In addition, the presence of clothing may influence the severity of the burn, while the thickness of the epidermis is known to also play an important role; there is a wide range of epidermal thicknesses in different locations of the human body and, in particular, geriatric and younger patients may be more susceptible to burns because of a generally thinner epidermal layer in their skin. Using a combined experimental and computer simulation methodology we hope to investigate these physical factors, and determine their relative importance.

There has recently been a lot of attention on the role of scalding in paediatrics, with younger children possessing relatively thin epidermal layers of skin. Young children are also more susceptible to soup burns as they often reach for hot food or beverages that are on elevated surfaces, and accidentally tip the fluids over themselves (Drago, 2005). For example, children reaching up to remove hot food from microwaves or pans from the top of stoves are obviously very dangerous. Noodle soups, which can be popular with children, have been identified as potentially one of the most dangerous fluids for paediatric scalding, because of the ability of these soups to stay hotter longer (Shalom et al., 2007). Cooling can be slowed in hot soups because the thicker soups can exhibit 
less convection and a dry skin can form on the top of the fluid reducing evaporative cooling. Furthermore, ready-to-eat noodle soups, are often packaged in unstable containers that are tall and have small bases (Greenhalgh et al., 2006). This has led to a sizable number of paediatric burns as children often tip over these unstable containers.

While geriatric patients may have received less attention than young children, in terms of the dangers of scalding, they can be both more susceptible and more incapacitated by scalds. This is of growing concern considering our elderly population form an increasingly large proportion of our patients. Burn injuries can be particularly debilitating as geriatric patients may already be burdened with comorbidities, and require a quick resolution to their injuries in order to maintain a higher functioning lifestyle (Lewandowski et al., 1993; Dissanaike and Rahimi, 2009). Older patients are also believed to suffer the physical and emotional scars to a much greater degree than younger patients (Engrav et al., 1987; Rossignol et al., 1985; Davidge and Fish, 2008). Elderly patients generally have weaker immune systems, with burn wound sepsis one of the possible complications that can arise, and the wounds typically heal much slower (Lewandowski et al., 1993; Le et al. 1986). The slower recovery times exhibited by geriatric patients can require more in-hospital rehabilitation or aftercare, and have adverse effects on the quality of life outcomes of elderly patients (Davidge and Fish, 2008). Understanding and preventing scald burns in this group, therefore, is important but also challenging, as the physical characteristics of the skin deteriorate with age; becoming thinner, wrinkled, fragile and dry. Elderly patients may also have decreased pain perception and tactile sensitivity, especially peripheral sensation, which can lead to a greater incidence and severity of burn (Morey, 2007). However, it is the decreases in epidermal thickness and how this might render the skin more susceptible to burn damage that is the focus of this study (Sauermann et al., 2002; Gilchrest, 1996).

Establishing guidelines for burn prevention and promoting education has become of paramount importance when limiting burn injuries; in particular, spreading the message to reduce temperatures in hot water heaters has dramatically reduced the incidence of these scald burns (Huyer and Corkum, 1997). Here, we consider the physical factors that might influence soup burns, such as the role that the soups temperature, viscosity and solid phases play in causing a soup burn. The physical properties of the substrate must also be evaluated including, the presence or absence of clothing, and the thickness of the epidermal layer. We investigate the properties of the soup that might affect the severity of a skin burn, in the hope of further educating the parents of younger children and caregivers of elderly people on both the dangers of scald burns from hot soups, and identifying any potential safeguards to prevent such burns.

In order to capture the heat transfer into a patient's skin as a consequence of exposure to hot soups we combine an experimental analysis of the thermodynamics and fluid mechanics of soup spills with a numerical analysis of heat transfer through the skin. In particular, in elucidating the effects of the physical properties of the soup on scald severity, we contrast hot water with the two most popular soups in the US: Campbell's Tomato Soup (which is a viscous but still predominantly homogeneous fluid) and Campbell's Chicken Noodle Soup (which is both viscous and heterogeneous, consisting of more discrete food chunks). These fluids are poured down a physical representation of the skin to capture the fluids thermal and hydrodynamic behaviour in this setting. The computational analysis of subsequent heat transfer through the surface of body tissue involves the solution of Pennes bioheat equation; a heat transfer model which takes into 
account tissue structure, metabolism and blood supply (Pennes, 1948; Wilson and Spence, 1988). This equation can easily be solved numerically using a finite difference method (Wilson and Spence, 1988; Ng and Chua, 2002), finite element method (Ng and Chua, 2002; Baldwin et al., 2012), boundary element method (Majchrzak and Jasinski, 2003; Ng et al., 2010) spectral method (Dehghan and Sabouri, 2012), or for relatively simple systems, solved analytically (Shih et al., 2007). The damage rate can be described using a standard Arrhenius model, with the damage function of Henriques and Moritz commonly used to model the severity of the burn (Moritz and Henriques, 1947). Here, as mentioned above, we also use a physical model of the skin to measure the surface temperature as various fluids are poured over the surface of the skin. The temperatures from our experimental analysis then serve as the input to our numerical analysis, where we calculate the heat that is transferred into the skin and predict the damage that occurs within the skin layers. In other words, we combine an experimental analysis of soup flow over a physical representation of the skin with a computational heat transfer model to predict the severity of a burn as a consequence of a particular soup scald, directly accounting for both the viscosity and solid constituents in the soup. Our methodology is detailed in the next section.

\section{Materials and methods}

The severity of a burn is predicted through a combination of experimental and numerical means. The experimental part uses a physical model of the skin and underlying tissue. The hot fluid is poured over this physical model and the surface temperature is measured as a function of time. Measuring the internal temperature experimentally is difficult, but it is the elevation of internal temperatures which is responsible for the severity of the burn. Therefore, to capture these internal temperatures the results from the experimental part of our model serve as input to the numerical part of our model. The numerical part of our model takes the time-dependent surface temperatures as its boundary conditions and numerically captures the heat flux through the skin and tissue. This enables us to predict the degree of damage that the skin sustains as a consequence of the different fluids as they flow over the skin or clothing.

Our approach integrates a physical model with a numerical model of the skin (Loller et al., 2015). The physical model consists of two plastic layers with thermal properties that are close to that of real skin. The experimental setup from which we conducted tests using water, tomato soup and chicken noodle soup, consists of a thin $0.762 \mathrm{~mm}$ layer of polycarbonate at the surface (with a thermal conductivity of $0.205 \mathrm{~W} \mathrm{~m}^{-1} \mathrm{~K}^{-1}$, closely matching the thermal conductivity of the epidermal layer of the skin), and a thick layer of low density polyethylene underneath (with a thermal conductivity of $0.33 \mathrm{~W} \mathrm{~m}^{-1} \mathrm{~K}^{-1}$, closely matching the thermal conductivity of the dermal layers of the skin). These two polymers were adhered to each other using thermal epoxy and a thermocouple was also adhered at the top surface of the polycarbonate layer using thermal epoxy. We poured the water and soups down the physical model of the skin and recorded the temperature at the surface of the skin as a function of time; this temperature then served as the input into our numerical model. 
We compared plain water, Campbell's condensed tomato soup and Campbell's condensed chicken noodle soup (the two most popular soups in the US) (CNN iReport, 2016). When testing the two soups, we mixed one can of the condensed soup with one can of water, as described in the cooking directions. When testing the water, we simply considered a volume of water equivalent to two cans of soup (each can is $290 \mathrm{~mL}$ ). We then microwaved the fluids on high for three minutes, allowed the fluid to sit for one minute, and stirred the fluid (following the cooking instructions for the soups). The fluid was immediately poured down the experimental setup of the skin, which was placed on a $20^{\circ}$ inclined plane.

The temperature at the surface of the physical model as a function of time, when plain water, tomato soup and chicken noodle soup were poured down the surface, are depicted in Figure 1. In particular, the relative temperature (the temperature at the surface of the skin divided by the maximum temperature) is depicted as a function of time. This allows us to better compare the effects of the different fluids which may possess slightly different temperatures subsequent to the heating process; while the water was brought to a boil, the thicker soups reached slightly lower temperatures of $95^{\circ} \mathrm{C}$ and $86^{\circ} \mathrm{C}$, for the tomato and chicken noodle soups, respectively. We will discuss this data further in the results section. For now, we turn our attention to how the dermal temperature throughout the skin layers is determined from the skin surface temperature. In particular, the results from our fluid and heat transfer experiments are fed into a numerical model of the heat transfer through the skin, to estimate the amount of damage sustained by the heated fluids.

Figure 1 The relative temperatures at the surface of a physical model of the skin (a thin polycarbonate layer on top of a low-density polyethylene layer) as a function of time (see online version for colours)

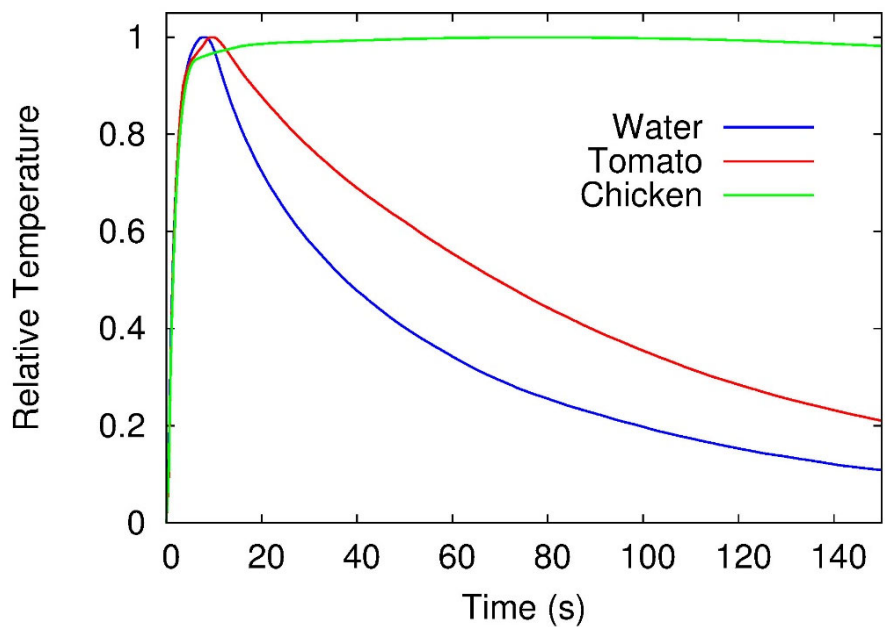

Notes: The relative temperature is defined as $\left(T-T_{o}\right) /\left(T_{\max }-T_{o}\right)$, where $T$ is temperature, $T_{\max }$ is the maximum temperature measured at the surface and $T_{o}$ is the ambient temperature. Three fluids are contrasted; hot water, tomato soup and chicken noodle soup. 
Table 1 Nomenclature

\begin{tabular}{|c|c|}
\hline \multirow[t]{4}{*}{ Epidermis layer } & $d_{e}=0.1 \mathrm{~mm}$ \\
\hline & $k_{e}=0.21 \mathrm{~W} /(\mathrm{m} \mathrm{K})$ \\
\hline & $c_{e}=3.6 \times 10^{3} \mathrm{~J} /(\mathrm{kg} \mathrm{K})$ \\
\hline & $\rho_{e}=1.2 \times 10^{3} \mathrm{~kg} / \mathrm{m}^{3}$ \\
\hline \multirow[t]{4}{*}{ Dermis layer } & $k_{d}=0.21 \mathrm{~W} /(\mathrm{m} \mathrm{K})$ \\
\hline & $c_{d}=3.6 \times 10^{3} \mathrm{~J} /(\mathrm{kg} \mathrm{K})$ \\
\hline & $\rho_{d}=1.2 \times 10^{3} \mathrm{~kg} / \mathrm{m} 3$ \\
\hline & $q_{d}=200 \mathrm{~W} / \mathrm{m}^{3}$ \\
\hline \multirow[t]{2}{*}{ Upper dermis layer } & $d_{u d}=0.6 \mathrm{~mm}$ \\
\hline & $B_{u d}=2 \mathrm{~kg} /\left(\mathrm{m}^{3} \mathrm{~s}\right)$ \\
\hline \multirow[t]{2}{*}{ Lower dermis layer } & $d_{l d}=0.5 \mathrm{~mm}$ \\
\hline & $B_{l d}=2 \mathrm{~kg} /\left(\mathrm{m}^{3} \mathrm{~s}\right)$ \\
\hline \multirow[t]{6}{*}{ Fat layer } & $d_{f}=2 \mathrm{~mm}$ \\
\hline & $k_{f}=0.16 \mathrm{~W} /(\mathrm{m} \mathrm{K})$ \\
\hline & $c_{f}=2.3 \times 10^{3} \mathrm{~J} /(\mathrm{kg} \mathrm{K})$ \\
\hline & $\rho_{f}=0.85 \times 10^{3} \mathrm{~kg} / \mathrm{m}^{3}$ \\
\hline & $q_{f}=5 \mathrm{~W} / \mathrm{m}^{3}$ \\
\hline & $B_{f}=0.6 \mathrm{~kg} /\left(\mathrm{m}^{3} \mathrm{~s}\right)$ \\
\hline \multirow[t]{6}{*}{ Muscle layer } & $d_{m}=6.8 \mathrm{~mm}$ \\
\hline & $k_{m}=0.53 \mathrm{~W} /(\mathrm{m} \mathrm{K})$ \\
\hline & $c_{m}=3.8 \times 10^{3} \mathrm{~J} /(\mathrm{kg} \mathrm{K})$ \\
\hline & $\rho_{m}=1.27 \times 10^{3} \mathrm{~kg} / \mathrm{m} 3$ \\
\hline & $q_{m}=800 \mathrm{~W} / \mathrm{m}^{3}$ \\
\hline & $\omega_{m}=0.5 \mathrm{~kg} /\left(\mathrm{m}^{3} \mathrm{~s}\right)$ \\
\hline Specific heat of blood & $c_{b}=3.8 \times 10^{3} \mathrm{~J} /(\mathrm{kg} \mathrm{K})$ \\
\hline Ambient temperature of blood & $T_{a}=35.5^{\circ} \mathrm{C}$ \\
\hline
\end{tabular}

We began by determining the solution to the Pennes bioheat equation, the most popular model for the heat transfer through the skin and subcutaneous tissue. This model also accurately captures the physiological effects of the skin and underlying tissue. The Pennes (1948) bioheat equation is given by:

$$
\rho c \frac{\partial T}{\partial t}=\nabla k \nabla T+\omega c_{b}\left(T_{a}-T\right)+q_{m}
$$

or in one dimension

$$
\rho c \frac{\partial T}{\partial t}=k \frac{\partial^{2} T}{\partial x^{2}}+\omega c_{b}\left(T_{a}-T\right)+q_{m}
$$

where $\rho$ is the density of the tissue, $c$ is the specific heat of the tissue, $k$ is the thermal conductivity, $q_{m}$ is the local metabolic heating, $\omega$ is a measure of the perfusion of blood to the lower dermis layer, $c_{b}$ is the specific heat of blood, $T_{a}$ is the ambient temperature of blood, and $T$ is the local temperature within the skin, which we are predicting with the 
model. The Pennes bioheat equation is solved in one-dimension with the skin and underlying tissue represented through an epidermis layer, an upper dermis layer, a low dermis layer, a layer of fat and a layer of muscle. In particular, the physical characteristics of the different layers are taken from Wilson and Spence (1988) and the thicknesses of the upper dermal, lower dermal, fat and the total thickness in the current model are taken to be $0.6 \mathrm{~mm}, 0.5 \mathrm{~mm}, 2 \mathrm{~mm}$, and $4 \mathrm{~mm}$, respectively. The thickness of the epidermal layer is varied in our current study to determine the effect of thinner epidermal layers in geriatric patients. The perfusion of blood to the lower dermis is given by

$$
\omega=\frac{d_{u d} B_{u d}+d_{l d} B_{l d}+d_{f} B_{f}}{d_{l d}}
$$

where $d$ is the thickness of a layer, $B$ is the blood flow to the layer and the subscripts $u d$, $l d$, and $f$ represent the upper dermis, lower dermis, and fat, respectively. For completeness, the parameters from Wilson and Spence are reproduced in Table 1.

The Pennes bioheat equation is solved using a standard finite-difference approximation, to obtain the temperature as a function of time throughout the skin. Dirichlet boundary conditions are used, where the temperature within the body is set to the ambient temperature of blood, and the surface temperature is obtained from the experimental results depicted in Figure 1. In this way, we can determine the temperature profiles inside the skin as a consequence of the different fluids responsible for the scald burn. Furthermore, we use the Henriques integrals to predict the severity of the burns (Moritz and Henriques, 1947). The Henriques integrals are a simple Arrhenius model of damage accumulation of the form:

$$
I_{b}=\int_{0}^{t} P_{b} e^{\left(-\frac{\Delta E}{R T_{b}}\right)}
$$

and

$$
I_{d}=\int_{0}^{t} P_{d} e^{\left(-\frac{\Delta E}{R T_{d}}\right)}
$$

$\Delta E / R$ is the ratio of activation energy to universal gas constant. $P_{b}$ and $P_{d}$ are pre-exponential factors. $T_{b}$ and $T_{d}$ are the temperatures at the basal layer (the surface between epidermis and dermis layers) and dermal base (surface between dermis layer and subcutaneous tissue), respectively. The pre-exponential factors are zero if the temperature is insufficient to cause damage and a constant otherwise. These thresholds are given by:

$$
P_{b}= \begin{cases}0 & \text { if } T_{b}<44^{\circ} \mathrm{C} \\ 1.43 \times 10^{72} & \text { if } T_{b} \geq 44^{\circ} \mathrm{C}\end{cases}
$$

and

$$
P_{d}= \begin{cases}0 & \text { if } T_{d}<44^{\circ} \mathrm{C} \\ 2.86 \times 10^{69} & \text { if } T_{d} \geq 44^{\circ} \mathrm{C}\end{cases}
$$

When $I_{b}$ is greater than 0.53 a first degree burn is predicted, when $I_{b}$ is greater than one then a second degree burn is predicted, and when $I_{d}$ is greater than one then a third degree 
burn is predicted. The Henrique integrals are calculated as a function of time from the simulation data, and the time taken for a given severity of burn to occur can be numerically predicted as a direct consequence of the experimental temperature at the surface of the skin. The Henriques integrals do not include thermoregulatory effects, which may come into play over larger time scales. However, the Henrique integrals have been widely used in the literature, and we use them here under the assumption that thermoregulatory effects will not affect the degree of burn over the short timeframe that the soup is in contact with the skin; the thermoregulatory effects will, however, influence the body's recovery from the scald burn which is outside the scope of this study. In the next section we consider the results from our experiments and subsequent numerical analysis.

\section{Results and discussion}

As mentioned earlier, the temperature at the surface of the experimental setup is depicted in Figure 1. In particular, the relative temperature (the temperature at the surface of the skin divided by the maximum temperature) is depicted as a function of time. The data is averaged over ten occurrences and the standard deviations in the data were always less than $3 \%$ of the average values (not shown in Figure 1). The surface of the skin reaches its maximum temperature within a few seconds after having the water poured over the surface, and the heat is readily transferred from the water to the surface. However, the low viscosity of the water means that the water flows down the inclined slope very quickly (which we would also expect for other hot beverages, like coffee or tea). The tomato soup, on the other hand, is more viscous, or thicker, than the water and flows down the inclined slope much slower. This results in the temperature at the surface of the skin being elevated for longer periods of time. In sharp contrast to both of these fluids is the chicken noodle soup. The chicken noodle soup contains a large amount of solid constituents; in other words, there are solid noodles and pieces of food suspended in the fluid. This has a dramatic effect on the fluid properties of the soup, as can be seen in Figure 1. While the hot water and tomato soup reach a maximum temperature early on, and then decrease quite rapidly as the fluids flow down the surface, the chicken noodle soup causes a sustained elevated temperature at the surface of the skin. The solid constituents of the soup clump together on the surface of the skin and therefore the flow of the soup is hindered in certain regions as it flows down the inclined plane. As a result, the hot chicken noodle soup remains in contact with the surface of the skin for longer time periods in the regions where there is an aggregate of solid pieces of soup on the surface. We now turn our attention to the numerical analysis in order to predict the severity of the skin burn as a consequence of these experimental temperature profiles.

The simulation size is $4,000 \Delta x$, where $\Delta x$ is $1 \mu \mathrm{m}$. The thicknesses of the dermis and fat layer are taken from Wilson and Spence while the thickness of the epidermal layer is varied from $30 \mu \mathrm{m}$ to $150 \mu \mathrm{m}$. While this might reflect the different thicknesses of epidermal layers at different regions of the body, it also elucidates the effects of reduced skin thickness in geriatric or paediatric patients. The time step in the simulations, $\Delta t$, is 1 $\mu \mathrm{s}$. We consider the time taken for first, second and third degree burns to occur as a 
function of temperature, for different epidermal thicknesses, in Figures 2 to 4 . In particular, Figure 2 depicts the results for the simulations where water is poured over the skin, Figure 3 the results for tomato soup and Figure 4 the results for chicken noodle soup. For all systems considered here, as the temperature of the fluid is increased, or the thickness of the epidermal layer is decreased, the time for a burn to occur decreases, as we might expect. As the temperatures are reduced, the times increase rapidly until we do not observe any occurrence of a burn for lower temperature conditions.

Figure 2 Simulation results for systems when water is poured over the skin. The time taken, after the spill occurs for, (a) a first degree burn (b) a second degree burn (c) a third degree burn to occur are depicted as a function of the initial temperature of the water and for different epidermal thicknesses (see online version for colours)

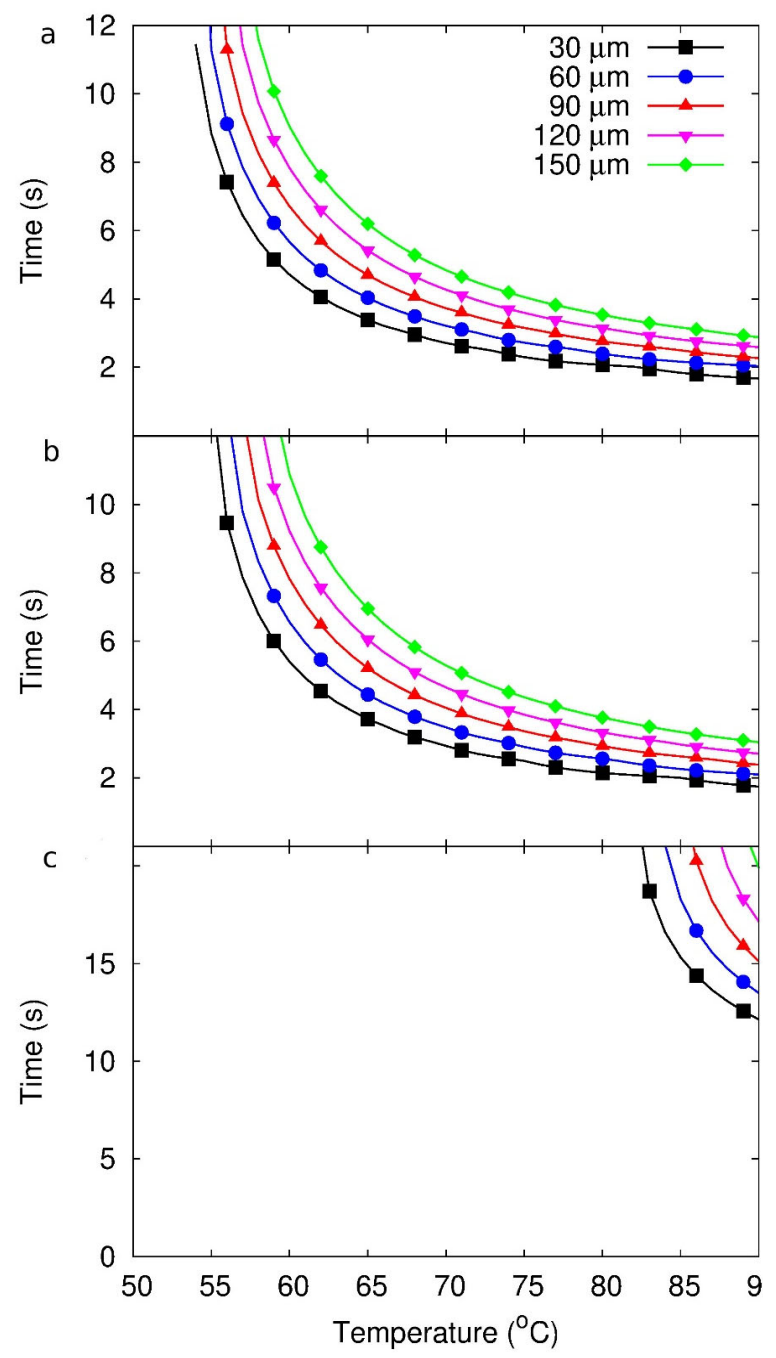


Figure 3 Simulation results for systems when tomato soup is poured over the skin. The time taken, after the spill occurs for, (a) a first degree burn (b) a second degree burn (c) a third degree burn to occur are depicted as a function of the initial temperature of the tomato soup and for different epidermal thicknesses (see online version for colours)

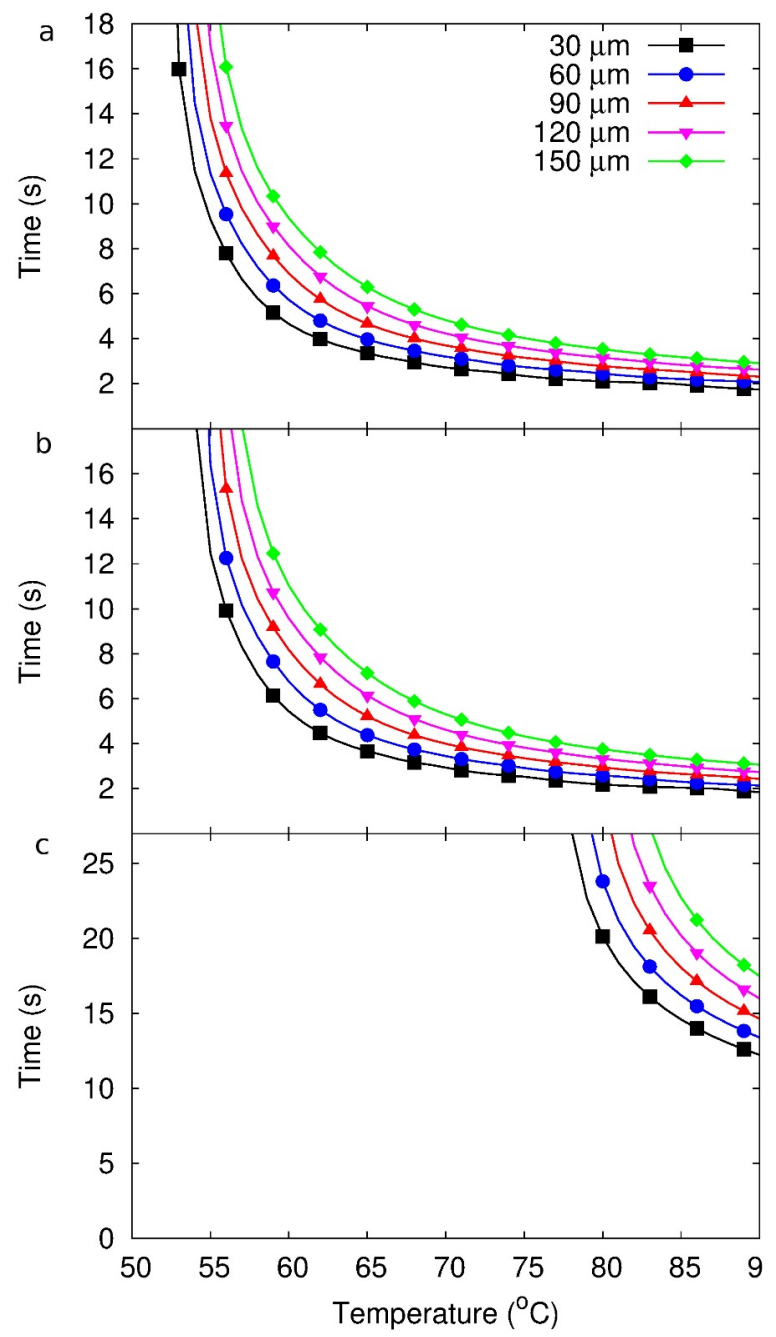

The time taken for a first degree burn to occur as a consequence of a hot water spill is shown in Figure 2(a). For temperatures as low as approximately $58^{\circ} \mathrm{C}$ we predict that a first degree burn could occur within ten seconds for the thicker epidermal layers, but within half this time for thinner epidermal layers. For hotter temperatures, the time taken for a first degree burn to occur in thicker skin thicknesses is around three seconds, while burns occur in skin with a thinner epidermal layer in around two seconds. Figure 2(b) shows the same system, but we now consider the time taken for a second degree burn to occur. The curves are shifted slightly to longer times and higher temperatures, but there is little difference between the predicted time for first degree burn to occur and a second degree burn to occur in the systems considered here. However, the times and temperatures required for a third degree burn to occur when hot water is poured over the 
skin are significantly different. Figure 2(c) shows that in order to observe a third degree burn from hot water, the temperature of the water must exceed $82^{\circ} \mathrm{C}$ and this requires that no intervention occur within this 12 second period (such as applying cold water).

Figure 4 Simulation results for systems when chicken noodle soup is poured over the skin. The time taken, after the spill occurs for, (a) a first degree burn (b) a second degree burn (c) a third degree burn, to occur are depicted as a function of the initial temperature of the chicken noodle soup and for different epidermal thicknesses (see online version for colours)

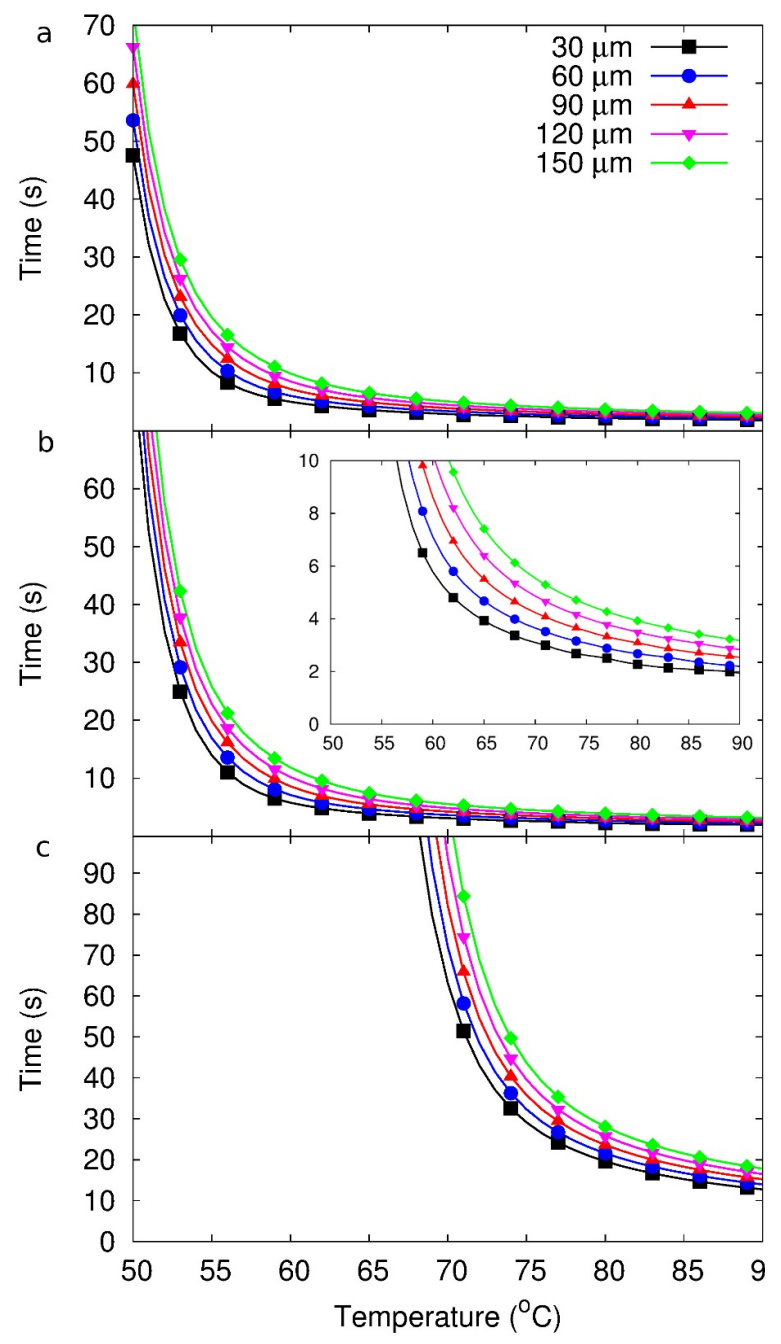

The effects of pouring tomato soup over the skin are considered in Figure 3. The results are similar to the systems considering water, depicted in Figure 2, with the main differences being a shift in the curves such that the time over which burns occur are increased and the temperature at which burns can occur are now slightly lower. This is expected as the more viscous tomato soup flows down the surface slower, and the hot soup remains in contact with the skin for longer periods of time. The effects of having 
thinner epidermal layers are still more pronounced at lower temperatures, as we found for the water burns. For example, for tomato soup at $90^{\circ} \mathrm{C}$ the time for a first, second degree or third degree burn to occur is 1.7 seconds, 1.85 seconds and 12.2 seconds, respectively, for skin with an epidermal layer of $30 \mu \mathrm{m}$. With an epidermal layer of $150 \mu \mathrm{m}$, the time for a first, second or third degree burn is around 2.9 seconds, 3.1 seconds and 17.5 seconds, respectively. Contrast this to the situation were the soup is at a lower temperature. The time for a given degree of burn to occur is much more sensitive to the epidermal thickness, and for Tomato soup at $57^{\circ} \mathrm{C}$, the predicted time for a second degree burn to occur drops from 17.9 seconds to 8 seconds as the thickness of the epidermal layer is reduced from $150 \mu \mathrm{m}$ to $30 \mu \mathrm{m}$. A fact that is expected to play a large role in the severity of soup burns in geriatric and paediatric patients.

Figure 5 The time for a second degree burn to occur as a function of the temperature of the hot water poured down the surface of the skin (see online version for colours)

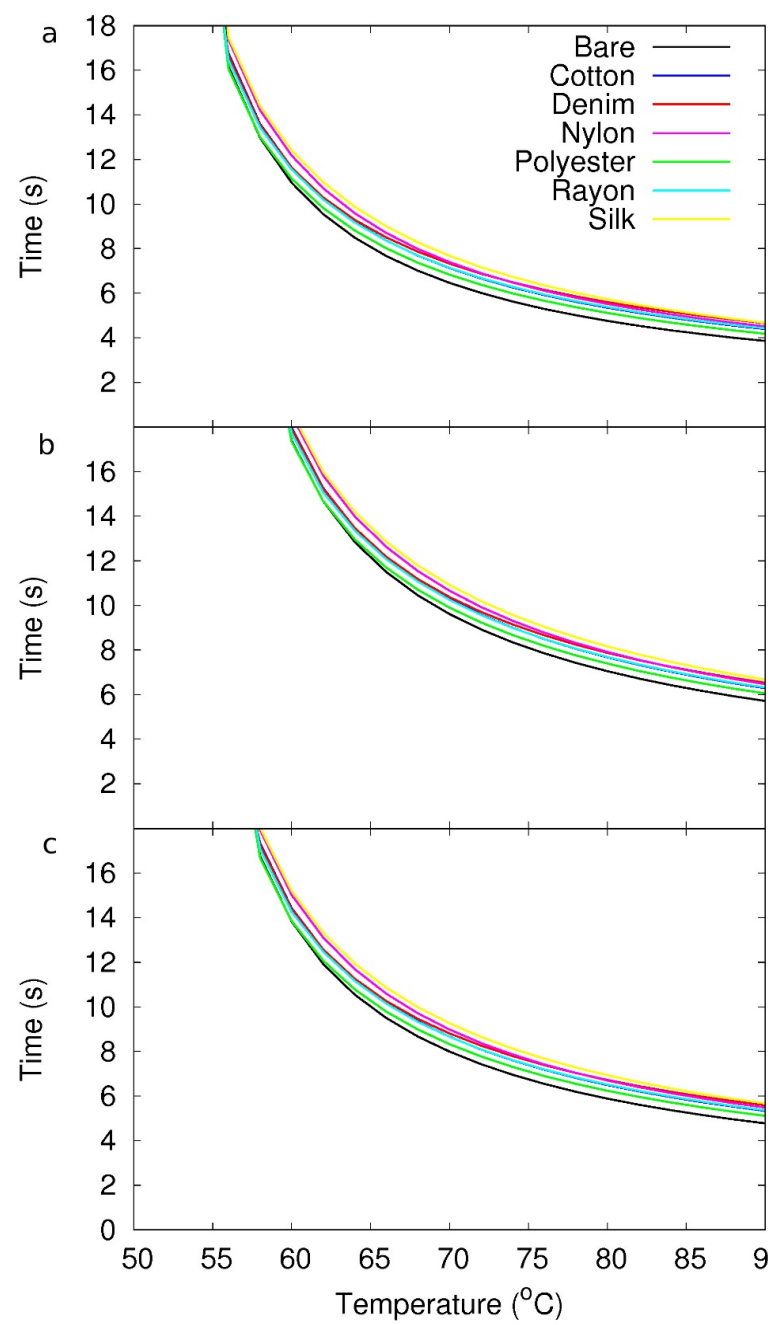

Notes: Figures 5(a), 5(b) and 5(c) depict the systems with epidermal layer thicknesses of $30 \mu \mathrm{m}, 90 \mu \mathrm{m}$ and $150 \mu \mathrm{m}$, respectively. 
The severity of a burn with chicken noodle soup is predicted to be much more severe than that of the water and tomato soup burns. For example, even for chicken noodle soup at a very low temperature of $50^{\circ} \mathrm{C}$ we predict that first and second degree burns can occur after around 48 seconds and 70 seconds, respectively, and even at temperatures as low as $67^{\circ} \mathrm{C}$ a third degree burn can occur after 100 seconds; although after such long times we would reasonably expect that the hot soup would be removed from the surface of the skin. For higher temperatures the time for a given burn to occur are comparable for all liquids considered here. For example, the inset of Figure 4(b) shows the same data as in Figure 4(b), but over a reduced range of times to better compare with the other fluids (which had little effect for long time periods as the fluid would have flowed from the surface). The time for a second degree burn to occur with a fluid of $90^{\circ} \mathrm{C}$ ranges from 2 to 3 seconds as a consequence of epidermal thickness, regardless of the type of fluid causing the burn. The effects of increased viscosity and the greater presence of solid constituents, for soups like the chicken noodle soup, would therefore appear to be more pronounced at intermediate temperatures. In particular, the temperature range of around $70^{\circ} \mathrm{C}$ or $75^{\circ} \mathrm{C}$, typical temperatures at which soups might be served, are where we see the greatest effects for the systems considered here. For example, in this temperature range no third degree burns were predicted for the water or tomato soup scalds, while for the chicken noodle soups a third degree burn could occur, albeit after quite long times.

The effects of clothing were also considered, in particular, we took pieces of different fabric (cotton, denim, nylon, polyester, rayon and silk) and performed the same analysis as above by pouring hot water over the fabric covered artificial skin. Our reasoning was that the longer the soups are in contact with the skin the more sever one might expect the severity of the scald, therefore fabric which would absorb to some extent the hot fluid might also increase the severity of the scald. However, what we found was the opposite. Figure 5 shows the time for a second degree burn to occur as a function of the temperature of the hot water poured down the surface of the skin. Figures 5(a), 5(b) and 5(c) depict the systems with epidermal layer thicknesses of $30 \mu \mathrm{m}, 90 \mu \mathrm{m}$ and $150 \mu \mathrm{m}$, respectively. However, the most obvious result is that the presence of the fabric had very little effect. The fabric raised the time before a burn occurred and appears to slightly protect the skin. Therefore, rather than increasing the severity of a scald, we predict that the presence of clothing will slightly reduce the severity of the burn.

\section{Discussion and conclusions}

The combination of experimental data, and the numerical solution of the bioheat equation, led to a methodology that could be used to predict the severity of scalds as a consequence of accidental soup spills. The results from the simulations show that the most severe burns occur over the longest exposure times, with the chicken noodle soup providing the most severe burns, followed by the tomato soup and then by the water. The viscosity, and therefore contact time between the fluid and the skin, plays a substantial role in this conclusion. Furthermore, the presence of solid constituents in the chicken noodle soup, in particular, prevents the soup from flowing from the skin, which increases the skin's exposure time to the elevated temperatures of the soup, and therefore can significantly increase the severity of a burn. 
Soups are often considered a low-cost, nutritional, and easily prepared food type. This ease of preparation makes soups a popular food choice for care givers of young and elderly people. Combined with the reduced epidermal thickness in paediatric and geriatric patients, this makes soup burns potentially more dangerous to the most vulnerable people. Caregivers and parents may not be aware that different types of soups can result in more or less severe scalds, depending on properties such as viscosity and the presence of solid constituents in the soup. Healthcare providers should advocate for appropriate soup temperatures in restaurants, while also providing education to parents and caregivers who prepare meals.

\section{References}

Baldwin, A., Xu, J. and Attinger, D. (2012) 'How to cool a burn: a heat transfer point of view', J. Burn. Care Res., Vol. 33, No. 2, pp.176-187.

CNN iReport (2016) What is The Most Popular Soup in The US? [online] http://ireport.cnn.com/ docs/DOC-1123167 (accessed 20 August 2016).

Davidge, K. and Fish, J. (2008) 'Older adults and burns', Geriatr. Aging., Vol. 11, No. 5, pp.270-275.

Dehghan, M. and Sabouri, M. (2012) 'A spectral element method for solving the Pennes bioheat transfer equation by using triangular and quadrilateral elements', Appl. Math Model, Vol. 36, No. 12, pp.6031-6049.

Dissanaike, S. and Rahimi, M. (2009) 'Epidemiology of burn injuries: highlighting cultural and socio-demographic aspects', Int. Review Psych., Vol. 21, No. 6, pp.505-511.

Drago, D.A. (2005) 'Kitchen scalds and thermal burns in children five years and younger', Pediatrics, Vol. 115, No. 1, pp.10-16.

Engrav, L.H., Covey, M.H., Dutcher, K.D., Heimbach, D.M., Walkinshaw, M.D. and Marvin, J.A. (1987) 'Impairment, time out of school, and time off from work after burns', Plast. Reconstr. Surg., Vol. 79, No. 6, pp.927-932.

Gilchrest, B.A. (1996) 'A review of skin ageing and its medical therapy', Brit. J. Dermatol., Vol. 135, No. 6, pp.867-875.

Greenhalgh, D.G., Bridges, P., Coombs, E., Chapyak, D., Doyle, W., O’Mara, M.S. and Palmieri, T.L. (2006) 'Instant cup of soup: design flaws increase risk of burns', J. Burn Care Res., Vol. 27, No. 4, pp.476-481.

Huyer, D.W. and Corkum, S.H. (1997) 'Reducing the incidence of tap-water scalds: strategies for physicians', Can. Med. Assoc. J., Vol. 156, No. 6, pp.841-844.

Le, H.Q., Zamboni, W., Eriksson, E. and Baldwin, J. (1986) 'Burns in patients under 2 and over 70 years of age', Ann. Plast. Surg., Vol. 17, No. 1, pp.39-44.

Lewandowski, R., Pegg, S., Fortier, K. and Skimmings, A. (1993) 'Burn injuries in the elderly', Burns, Vol. 19, No. 6, pp.513-515.

Loller, C., Buxton, G.A. and Kerzmann, T.L. (2015) 'Hot soup! Correlating the severity of liquid scald burns to fluid and biomedical properties', Burns, Vol. 42, No. 3, pp.589-597.

Majchrzak, E. and Jasinski, M. (2003) 'Numerical estimation of burn degree of skin tissue using the sensitivity analysis methods', Acta Bioeng. Biomech., Vol. 5, No. 1, pp.93-108.

Morey, P. (2007) 'Skin tears: a literature review', Prim Inten., Vol. 15, No. 3, p.122.

Moritz, A.R. and Henriques, F.C. (1947) 'The relative importance of time and surface temperature in the causation of cutaneous burns. Studies of thermal injury II', Am. J. Path., Vol. 23, No. 5, p.659.

Ng, E.Y. and Chua, L.T. (2002) 'Prediction of skin burn injury. Part 2: parametric and sensitivity analysis', Proc. Inst. Mech. Eng. H., Vol. 216, No. 3, pp.171-183. 
Ng, E.Y., Tan, H.M. and Ooi, E.H. (2010) 'Prediction and parametric analysis of thermal profiles within heated human skin using the boundary element method', Phil. Trans. R. Soc. A., Vol. 368, No. 1912, pp.655-678.

Pennes, H.H. (1948) 'Analysis of tissue and arterial blood temperatures in the resting human forearm', J. Appl. Physiol., Vol. 1, No. 2, pp.93-122.

Rossignol, A.M., Locke, J.A., Boyle, C.M. and Burke, J.F. (1985) 'Consumer products and hospitalized burn injuries among elderly Massachusetts residents', J. Am. Geriatric. Soc., 1 November, Vol. 33, No. 11, pp.768-772.

Sauermann, K., Clemann, S., Jaspers, S., Gambichler, T., Altmeyer, P., Hoffmann, K. and Ennen, J. (2002) 'Age related changes of human skin investigated with histometric measurements by confocal laser scanning microscopy in Vivo', Skin Res Technol., Vol. 8, No. 1, pp.52-56.

Shalom, A., Bryant, A., Smith-Meek, M., Parsons, L.R. and Munster, A. (2007) 'Noodles stay hotter longer', J. Burn Care Res., Vol. 28, No. 3, pp.474-477.

Shih, T.C., Yuan, P., Lin, W.L. and Kou, H.S. (2007) 'Analytical analysis of the Pennes bioheat transfer equation with sinusoidal heat flux condition on skin surface', Med. Eng. Phys., Vol. 29, No. 9, pp.946-953.

Wilson, S.B. and Spence, V.A. (1988) 'A tissue heat transfer model for relating dynamic skin temperature changes to physiological parameters', Phys. Med. Biol., Vol. 33, No. 8, p.895. 